\title{
Stem cell and toxicology
}

Keywords: Stem cell; Toxicology; Bone marrow transplants; Multipotent stem cells; Adipose tissue

\section{Introduction}

Undifferentiated cells were initially utilized as a part of bone marrow transplants in 1956. Since the late 1990's when embryonic undifferentiated cells were found in people, the advances in undeveloped cell science have been rapid. However a significant part of the work in the field of stem cell science is centered around comprehension ordinary advancement and disarranges of the human body and for toxicology screening (testing new medications). ${ }^{1}$

Multipotent stem cells could possibly help in the treatment of tissue that can't recover itself. This would be of advantage for spinal cord injuries and injuries to tactile organs, which also have likewise constrained repair abilities. Once more, research on these particular cell types can aid scientists in discovering treatment choices for different issue, not necessarily just using stem cells themselves to treat the disorder. ${ }^{2}$

\section{Background}

Stem cells are undifferentiated natural cells that can differentiate into particular cells and can divide (through mitosis) to deliver more stem cells. They are found in multicellular living beings. In warm blooded animals, there are two types of stem cells: embryonic cells, which are disengaged from the internal mass of blastocysts, and adult cells, which are found in different tissues. ${ }^{3}$

Stem cells in adult act as a repair framework for the body, renewing tissues. In embryo, stem cells can separate into all specific cells-ectoderm, endoderm and mesoderm. Additionally; keep up the typical turnover of regenerative organs, for example, blood, skin, or intestinal tissues. ${ }^{4}$

There are four known wellsprings of grown-up stem cells in people:

i. Bone marrow (commonly the femur or iliac crest).

ii. Adipose tissue (lipid cells), which requires extraction by liposuction.

iii. Blood, where blood is drawn and went through a machine that concentrates the stem cells and returns different parts of the blood to the donor.

iv. Stem cells can likewise be taken from umbilical cord blood soon after birth.

Occasionally; stem cells are used as a part of therapeutic treatment (e.g., bone marrow transplantation). They can now be isolated into particular cell sorts with characteristics dependable with cells of various tissues, for example, muscles or nerves. Embryonic cell lines and autologous embryonic cells created have additionally been proposed as promising possibility for future therapies. ${ }^{5}$

The classical properties of stem cells:

i. Self- reestablishment: the capacity to experience various cycles of cell division while keeping up the undifferentiated state.

\author{
Volume 2 Issue I - 2017 \\ Doaa Abdel Wahab El Morsi \\ Department of Forensic Medicine and Clinical Toxicology, \\ Mansoura Faculty of Medicine, Egypt
}

\begin{abstract}
Correspondence: Doaa Abdel Wahab El Morsi, Department of Forensic Medicine and Clinical Toxicology, Mansoura Faculty of Medicine, Egypt, Email Dr.Doaa20I4@outlook.com
\end{abstract}

Received: October 05, 2016 | Published: January II, 2017

ii. Potency: the ability to separate into specialized cell types. This requires stem cells to be either totipotent or pluripotent - to have the capacity to offer ascent to any full grown cell, although multipotent or unipotent progenitor cells are sometimes referred to as stem cells. Aside from this; it is said that stem cells is managed in a feedback system.

\section{Human stem cells as a new tool in toxicology}

Despite the fact that the stem cells could be used to assess the potential of any toxin/toxicant; it may be insightful examine some theoretical and practical matters related to our current state of understanding basic stem cell biology and their roles in normal development and any stem cell-related disease. ${ }^{6}$

Inspite the concept of stem cells has long been for quite some time a center of scholarly examination in the fields of embryology, developmental biology, plant biology, and cancer research. The detachment of human embryonic immature stem cells have brought stem cells into investigative/therapeutic groups. The hypothetical plausibility of applying human immature microorganisms for enhancing numerous human illnesses appeared to be conceivable before achieving any crucial comprehension of the perplexing variables which control an undeveloped cell's behavior. ${ }^{7}$

However, right now of attempting to distinguish the reasonable possibilities about the stem cells, endeavors are being made to apply immature cells for wide various applications, for example, for testing toxicity. To date, stem cells have been thought to be utilized for (1) essential comprehension of how stem cells manages the genome and its cell behaviour (cell multiplication in a symmetrical or asymmetrical form, separation, apoptosis, and so forth.), (2) regenerative medication or stem cell treatment, (3) drug discovery, (4) study poisoning of pharmaceuticals and stem cell treatment, (5) genetic treatment, and (6) the role in stem cell-derived sicknesses and in the aging procedure. It is difficult to look at the possibilities and a limitations of all these potential applications. ${ }^{8}$

Similarly essential, our comprehension of the pathogenesis of numerous human illnesss is not known. Thus, attempting to foresee the pathogenesis of a human disease (or how to counteract or cure the infection) by utilizing information, got from deficient toxicity testing, just depicts the insufficiencies of our present condition of knowledge. ${ }^{9}$ 


\section{Stem cell biology}

At the time of fertilization, the fertilized egg ("totipotent" stem cell), which contains genome of individual, should now confront the complex interactions of the external environment's affect on the pregnant female, of her genetic background, her nutritional status and diet, life style behavior, mental stress level, and of any medical treatments. From this dynamic association, the implantation of the totipotent stem cell begins a link of occasions to confer this cell to shape the embryonic or pluripotent stem cell. ${ }^{10}$

As these cells show up in the blastocyst, they, change the microenvironment leading to a further restriction of these pluripotent stem cells to become, multipotent cells that are committed to specific organ types. At last, progenitor stem cells, such as those seen in the lymphohematopoietic system appear. Transit-amplifying cells, or those derived from a stem cell are, somehow, committed to a life span. The terminally separated cells, such as a red blood cells or a lens cell, is limited from proliferating. ${ }^{11}$

Operationally, a stem cell is characterized as a cell that can isolate either symmetrically or asymmetrically after being given an appropriate external signal. Despite; the mechanism for controlling whether a stem cell divides, symmetrically or asymmetrically it is still unknown; some growth conditions do influence this cellular decision, including specific growth factors or $\mathrm{O} 2$ levels and antioxidants. ${ }^{12}$

\section{Nature of normal development, and health}

In utilizing human stem cells for potential poisoning; one must remember the dynamic nature of stem cell biology in vivo so that any in vitro result will not be misinterpreted to potential toxicities in vivo. The developmental process of the cells, involving an undifferentiated totipotent stem cell, with the total genetic information to produce an individual, includes numerous interactions and organization of molecules, biochemical reactions, organelle formation within cells, that regulate tissues and organisms, which form organ systems to create human being. All this occurs via unique environmental (physical, chemical, biological) interactions with specific genes. ${ }^{3}$

\section{Toxicity end points at the cell level}

At the cell level; exposure of cells to physical, chemical, biological agents, and even psychological stress will be translated into some response: The cancer problem is not a cell problem; rather it is a problem of cell interaction. But we must also remember that the integration of normal cells with the welfare of the whole organism is brought about by molecular messages acting on molecular receptors. ${ }^{9}$

It appears that three outcomes to this exposure will be (1) mutation, caused by either an error in DNA repair or by an error of DNA replication (genotoxicity), (2) cell death by necrosis or apoptosis (cytotoxicity), and (3) altered gene expression at the transcriptional, translational, or posttranslational levels (epigenetic toxicity). For genotoxicity and cytotoxicity assessments; various molecular and in vitro assays have been used and till now no consensus has been achieved. ${ }^{6}$

The consequences of these assays were (1) not reliable, (2) did not assess what they were assumed to measure, (3) extracted from cell types not relevant to the human system, (4) derived from the wrong conditions for measuring toxicities that occur in vivo at different conditions, and (5) in many cases, were misinterpreted. In vitro assays carry many limitations to measure "epigenetic toxicants" or, as some refer to them as "non genotoxicants". The endogenous - and exogenous induced intracellular signaling mechanisms were evolutionarily designed to regulate gene expression. ${ }^{13}$

Genetic or environmentally induced abnormal expression of genes is the basis of epigenetic toxicity. However, the recent microarray technologies, still leave much to be desired. Clearly, patterns of altered gene expression from exposed or diseased tissues can be reproduced and even have useful applications. However, extracting molecular expressed genes from normal, treated, or diseased tissue is extracting messages from a heterogeneous mixture of cell types. ${ }^{14}$

Moreover, some cells are stressed; others are dying of apoptosis, or mutated, and others are invading cells. So, the total message examined is the result of expression of all these types of cells. This point has been studied as they titled as: "Traditional microarrays"; which can neither distinguish between variations in gene expression resulting from an actual physiological change versus differences in cell-type frequency nor identify the contributions of different cell types to the total measured gene expression. ${ }^{15}$

In addition, ionizing and ultraviolet light radiation can produce chromosome and gene mutation, respectively, they can, induce oxidative stress, which, can altered gene expression. In effect, they can have epigenetic effects. It should also be stated that, at toxic doses, any agent (radiation, chemicals, biological) can be an indirect epigenetic toxicant, so the released substances from cell killing can act to stimulate the surviving cells to wound healing. ${ }^{16}$

In vivo toxicity involves the tissue's response to the exogenous chemical or therapeutic stem cell, as well as to the consequences of the chemical or stem cell products on the immune system. As any substance (chemical or stem cell product) enters the body, it will directly or indirectly interact with (1) the three types of cells (adult stem cells, transit amplifying cells, and the terminally differentiated cells) in the tissue and (2) with cells of the immune system. In both cases, intracellular signaling occurs. In the case of the cells of the immune system, various bioactive factors are secreted that can interact with the epithelial/endothelial cells that might have also been "primed" by the same toxicant. ${ }^{11}$

Study of how chemicals or stem cell products, could induce specific intracellular signaling to trigger epigenetic changes in vivo, will be a challenge. One of the characteristic properties of this class of agents is also to inhibit apoptosis, in that tumor promoting chemicals. ${ }^{9}$

In transplantation studies, it is noticed alteration in the apoptotic rate of cells. On contrary, if the agent causes upregulation of either cells of the immune system or cells of the affected tissues, cells could differentiate or apoptose. So; the stem cell itself will be subject to in vivo endogenous factors that could lead to symmetrical division (proliferation), induced asymmetrical division (differentiation, apoptosis), or senescence. ${ }^{7}$ These cells also can affect the immune response by secreted factors that could illicit a cellular response in the targeted tissue. This type of unexpected response has been reported. ${ }^{17}$

\section{The limitations of current in vitro assays}

To use in vitro mammalian cells, most assays have used either immortalized or cancer cells. Moreover, without an immunological or dynamic physiological environment, these abnormal could not predict how any of the three classes of normal human cells (the adult stem 
cells, the progenitor or terminally differentiated cells) would react in vivo. ${ }^{5}$

The different cell types (the few stem cells, the many progenitor and differentiated cells) is physiologically different. So, there will be a differential response depite the toxic potential of any agent. In fact, there is proof that these stem cells express drug transporter genes that pump out various classes of toxic chemicals. This might be one potential limitation for the use of pure stem cell populations to test for genotoxic chemicals. ${ }^{18}$

\section{Acknowledgements}

None.

\section{Conflict of interest}

The author declares no conflict of interest.

\section{References}

1. Cheng N, Wauthier E, Reid LM. Mature human hepatocytes from ex vivo differentiation of alginate-encapsulated hepatoblasts. Tissue Eng. 2008;14(1):1-7.

2. Shiraki N, Umeda K, Sakashita N, et al. Differentiation of mouse and human embryonic stem cells into hepatic lineages. Genes Cells. 2008;13(7):731-746.

3. Yu J, Hu K, Smuga Otto K, et al. Human induced pluripotent stem cells free of vector and transgene sequences. Science. 2009;324(5928):797801 .

4. Turpeinen M, Tolonen A, Chesne C, et al. Functional expression, inhibition and induction of CYP enzymes in HepaRG cells. Toxicol In Vitro. 2009;23(4):748-753.

5. Zhao S, Wehner R, Bornhäuser M, et al. Immunomodulatory properties of mesenchymal stromal cells and therapeutic consequences for immune-mediated disorders. Stem Cells Dev. 2010;19(5):607-614.

6. Basma H, Soto Gutiérrez A, Yannam GR, et al. Differentiation and transplantation of human embryonic stem cell-derived hepatocytes. Gastroenterology. 2009;136(3):990-999.
7. Zachar V, Prasad SM, Weli SC, et al. The effect of human embryonic stem cells (hESCs) long-term normoxic and hypoxic cultures on the maintenance of pluripotency. In Vitro Cell Dev Biol. 2010;46(34):276-283.

8. Warren L, Manos PD, Ahfeldt T, et al. Highly efficient reprogramming to pluripotency and directed differentiation of human cells with synthetic modified mRNA. Cell Stem Cell. 2010;7(5):618-630.

9. Voog J, Jones DL. Stem cells and the niche: a dynamic duo. Cell Stem Cell. 2010;6(2):103-115.

10. Van Dartel DA, Pennings JL, De la Fonteyne LJ, et al. Monitoring developmental toxicity in the embryonic stem cell test using differential gene expression of differentiation-related genes. Toxicol Sci. 2010;116(1):130-139.

11. Liu W, Deng Y, Liu Y, et al. Stem cell models for drug discovery and toxicology studies. Biochem Mol Toxicol. 2013;27(1):17-27.

12. Hodjat M, Rezvanfar MA, Abdollahi M. A systematic review on the role of environmental toxicants in stem cells aging. Food Chem Toxicol. 2015;86:298-308.

13. Asselin Labat ML, Vaillant F, Sheridan JM, et al. Control of mammary stem cell function by steroid hormone signaling. Nature. 2010;465(7299):798-802.

14. Rashid ST, Alexander GJ. Induced pluripotent stem cells: from Nobel Prizes to clinical applications. J Hepatol. 2013;58(3):625-629.

15. Chapin RE, Stedman DB. Endless possibilities: stem cells and the vision for toxicology testing in the $21^{\text {st }}$ century. Toxicol Sci. 2010;112(1):1722 .

16. Suter Dick L, Alves PM, Blaauboer BJ, et al. Stem cell-derived systems in toxicology assessment. Stem Cells Dev. 2015;24(11):1284-1296.

17. Niwa T, Tsukamoto T, Toyoda T, et al. Inflammatory processes triggered by Heliobacter pylori infection cause aberrant DNA methylation in gastric epithelial cells. Cancer Res. 2010;70(4):1430-1440.

18. Jannuzzi AT, Ozcagli E, Kovatsi L, et al. Using stem cells in toxicological assessments. Journal of Medical Toxicology and Clinical Forensic Medicine. 2016;2(1):1-2. 